\title{
DESENVOLVIMENTO, CRIAÇÃO E COMPORTAMENTO DE Toxorhynchites (Lynchiella) haemorrhoidalis haemorrhoidalis (Fabricius, 1794) (DIPTERA: CULICIDAE) NO LABORATÓRIO.
}

\author{
Rosa Sá Gomes Hutchings ${ }^{1}$
}

RESUMO - São descritos os comportamentos de predação, canibalismo e emergência de Toxorhynchites (Lynchiella) haemorrhoidalis haemorrhoidalis no laboratório. A duração do período de desenvolvimento de cada estágio é apresentada para larvas criadas no laboratório, sob diferentes condições de dieta, temperatura e luminosidade. As larvas de $T$. $h$. haemorrhoidalis, criadas com dieta de ração, completam seu desenvolvimento, apesar de demorarem mais para atingir o estágio adulto. A luminosidade não teve influência no tempo de desenvolvimento das larvas alimentadas com presas, ao contrário das alimentadas com ração. A baixa temperatura influenciou o desenvolvimento das larvas, independentemente da dieta, prolongando o período de desenvolvimento.

Palavras-chave: Toxorhynchites haemorrhoidalis, criação, desenvolvimento larval, comportamento, laboratório.

Development, breeding and behavior of Toxorhynchites (Lynchiella) haemorrhoidalis haemorrhoidalis (Fabricius, 1794) (Diptera: Culicidae) in the laboratory

ABSTRACT - The predation, canibalism and emergence behavior of Toxorhynchites (Lynchiella) haemorrhoidalis haemorrhoidalis in the laboratory is described. The development period for each instar, under laboratory conditions, is presented for rearing under different diet, temperature and light conditions. The larvae of $T . h$. haemorrhoidalis, reared with fish ration, completed their development, even though they took longer to achieve adult stage. Light conditions did not influence the development period of prey feeding larvae, contrary to ration fed larvae. Low temperature prolonged the larval development period, independent of diet.

Key words: Toxorhynchites haemorrhoidalis, breeding, larval development, behavior, laboratory

\section{INTRODUÇÃO}

As larvas dos mosquitos do gênero Toxorhynchites são predadoras de outras larvas de mosquitos. Os adultos, como não se alimentam de sangue, são inofensivos ao homem fazendo com que as espécies deste gênero sejam candidatas a controladores biológicos de outros mosquitos (STEFFAN, 1975; STEFFAN \& EVENHUIS, 1981). O possível uso de Toxorhynchites como controlador biológico de agentes vetores de doenças na região amazônica já foi discutido (HUTCHINGS, 1991). Para que qualquer programa de controle biológico tenha sucesso, a biologia do agente de controle tem que ser bem

1 Instituto Nacional de Pesquisas da Amazônia, Coordenação de Pesquisas em Entomologia, Caixa Postal 478, 69011-970, Manaus, Amazonas, Brasil. 
conhecida. Portanto, o estudo da bionomia no campo e no laboratório de Toxorhynchites

(Lynchiella) haemorrhoidalis haemorrhoidalis (Fabricius, 1794), espécie comum na região de Manaus, é de extrema importância para o desenvolvimento de métodos de controle biológico. Os criadouros naturais e os ciclos de desenvolvimento desta espécie no seu habitat natural são conhecidos (HUTCHINGS, 1990; HUTCHINGS, no prelo) e este trabalho objetiva complementar estas informaçoes com dados sobre o comportamento e desenvolvimento dos imaturos no laboratório. Para o desenvolvimento completo de uma larva de Toxorhynchites é necessário um grande número de presas, o que aumenta o custo de produção (TRIPS, 1979). Mediante o uso experimental da ração de peixe para criação de $T . h$. haemorrhoidalis se tenta avaliar a eficiência de utilização de uma dieta sem presas com um custo de produção reduzido.

Este trabalho tem como objetivos específicos: (1) Determinar se Toxorhynchites

(Lynchiella) haemorrhoidalis haemorrhoidalis pode ser criado em laboratório sobre diferentes condições de dieta, temperatura, e luminosidade; (2) Avaliar a influência das diferentes condições de criação sobre o desenvolvimento dos imaturos da espécie; e (3) Descrever o comportamento de predação, canibalismo e emergência desta espécie no laboratório.

\section{MATERIAL E MÉTODOS}

Os ovos de $T . h$ haemorrhoidalis e as larvas de outros culicídeos utilizados para as criações foram coletados, no ano de 1986, na sede do Instituto Nacional de Pesquisas da Amazônia (INPA) no Bairro do Coroado, Manaus, Amazonas. Estas coletas foram feitas em criadouros artificiais, tais como baldes, tambores, latas, pneus e tanques que acumulam água de chuva. As coletas no campus do INPA não obedeceram a um período rígido; sempre que necessário os criadouros existentes foram visitados, para coleta do material. Os ovos de Toxorhynchites foram colocados individualmente em copos plásticos descartáveis, brancos, de $7 \mathrm{~cm}$ de diâmetro de boca por $4,5 \mathrm{~cm}$ de altura, com água da torneira (aprox. $70 \mathrm{ml}$ ) e mantidos no laboratório numa temperatura entre $24^{\circ}$ e $30^{\circ} \mathrm{C}$ até à eclosão.

Para a manutenção dos Toxorhynchites até o estágio de pupa, as larvas foram alimentadas segundo duas dietas: larvas de outros culicídeos (presas; principalmente Culex spp.) ou uma ração à base de peixe (peixe seco moído). No caso de dieta com larvas, cada larva de $T$. $h$. haemorrhoidalis, recém-eclodida, recebeu como alimentação dez larvas de outros culicídeos. Após 24 horas, registrou-se o número de presas consumidas e, em seguida, completou-se a oferta de presas até dez larvas. $\mathrm{Na}$ dieta com ração, aproximadamente $1 \mathrm{mg}$ de ração foi colocada em cada copo ao eclodir 
a larva. A cada 24 horas a larva era retirada com ajuda de uma pipeta, a água era trocada, recolocando-se a larva junto com a mesma medida de ração. A troca da água era necessária dada à decomposição da ração não consumida.

Além das dietas, foram variadas também a luminosidade e a temperatura. Larvas recém-eclodidas foram equitativamente divididas em grupos, e cada grupo mantido em ambientes diferentes, tais como: sala de laboratório com luz artificial (temperatura entre $24-30^{\circ} \mathrm{C}$; denominado "laboratório"), estufa fechada sem luz (temperatura entre 28$31^{\circ} \mathrm{C}$; denominado "escuro") e sala de laboratório com luz ambiental (temperatura entre $22-26^{\circ} \mathrm{C}$; denominado "frio"). Em cada ambiente foram colocados dois grupos com a mesma quantidade de larvas; destes grupos, um foi criado com dieta de ração e outro com dieta de presas.

As pupas foram transferidas para gaiolas, até à emergência dos adultos. Os adultos foram mantidos com dieta de água açucarada $(10 \%)$, ou com uma mistura de água e glicose de milho, na mesma proporção, em algodão embebido e pendurado na parte superior da gaiola.

\section{RESULTADOS E DISCUSSÃO}

O período de incubação do ovo variou entre 24 e 72 horas. O período de incubação das outras espécies do gênero, normalmente varia desde 40 até 60 horas e depende da temperatura
(STEFFAN \& EVENHUIS, 1981).

Todos os estádios larvais do $T$. $h$. haemorrhoidalis são predadores de invertebrados ativos, principalmente larvas e pupas de outros culicídeos. As pupas são predadas quando há uma baixa ou nula concentração de larvas. As presas podem ser menores, ou até maiores, que o tamanho do predador. Nas criações de laboratório, tivemos a oportunidade de assistir a larvas de $2^{\circ}$ estádio de $T . h$. haemorrhoidalis predando larvas de $4^{\circ}$ estádio de Culex spp. As larvas de $T . h$. haemorrhoidalis comem as partes menos esclerotizadas, deixando apenas a cabeça da presa. Ao atingirem o $4^{\circ}$ estádio, quando em prépupa, as larvas de $T . h$. haemorrhoidalis, matam as presas, porém sem consumílas ou consomem apenas a metade delas. Este comportamento de predação e alimentação observado para $T, h$. haemorrhoidalis não é diferente daquele descrito por vários autores (CORBET \& GRIFFITHS, 1963; RUBIO et al., 1980; MACHADOALLISON, 1981; STEFFAN \& EVENHUIS, 1981) para outras espécies do gênero.

Em observações feitas durante as criações em grupos, no laboratório, registrou-se canibalismo por parte de $T$. $h$. haemorrhoidalis de todos os estádios mas, principalmente, por larvas de $4^{\circ}$ estádio. $O$ comportamento foi proporcionalmente menor na presença de outras presas, aumentando com a diminuição da disponibilidade de presas. Os registros de comportamento canibalístico entre larvas de 
Toxorhynchites, revisados por STEFFAN \& EVENHUIS (1981), indicam diferentes níveis de canibalismo para diferentes espécies de Toxorhynchites. O canibalismo observado em $T$. h. haemorrhoidalis é similar ao registrado para $T$. amboinensis (STEFFAN et al., 1980), no qual existe canibalismo em todos os estádios larvais.

Avaliamos o desenvolvimento larval de $T . h$. haemorrhoidalis, nos quatro estádios, em três ambientes diferentes e com dois tipos de dieta. Os resultados obtidos nestas criações estão resumidos na Tabela 1. Comparandose o tempo de desenvolvimento com as duas dietas, ração e presas, e somandose os resultados das criações feitas em ambientes diferentes, a duração média total do primeiro até o final do quarto estádio, para larvas de $T . h$. haemorrhoidalis alimentadas com presas, foi de $23.1 \pm 0.6$ dias e, para larvas alimıntadas com ração, de 32.5 \pm 0.8 dias. Esta diferença entre as duas dietas é muito significativa (teste-t NS (Nível de Significância) $1^{\circ} \Rightarrow 4^{\circ}$ est. $=$

Tabela 1. Duração média (dias) dos estádios larvais e estágio de pupa de Toxorhynchites $h$. haemorrhoidalis durante criações no laboratório sob condições diferentes de dieta, temperatura e luminosidade.

\begin{tabular}{|c|c|c|c|c|c|c|}
\hline & \multicolumn{6}{|c|}{ DURAÇĀO (dias) } \\
\hline & \multicolumn{2}{|c|}{$\begin{array}{l}\text { LABORATORIO: } \\
24^{\circ} \mathrm{C}-30^{\circ} \mathrm{C} \\
\text { COM LUZ }\end{array}$} & \multicolumn{2}{|c|}{$\begin{array}{l}\text { ESCURO: } \\
28^{\circ} \mathrm{C}-31^{\circ} \mathrm{C} \\
\text { SEM LUZ }\end{array}$} & \multicolumn{2}{|c|}{$\begin{array}{c}\text { FRIO: } \\
22^{\circ} \mathrm{C}-26^{\circ} \mathrm{C} \\
\text { COM LUZ }\end{array}$} \\
\hline & PRESA (Larva)* & RAÇĀO & PRESA (Larva) & RAÇĀO & PRESA (Larva) & RAÇÃO \\
\hline $\begin{array}{l}1^{0} \\
\text { Estádio }\end{array}$ & $\begin{array}{c}2.90(0.09) \\
{[115]}\end{array}$ & $\begin{array}{c}2.95(0.10) \\
{[96]}\end{array}$ & $\begin{array}{c}2.92(0.63) \\
{[12]}\end{array}$ & $\begin{array}{c}2.88(0.25) \\
{[18]}\end{array}$ & $\begin{array}{c}3.64(0.60) \\
{[11]}\end{array}$ & $\begin{array}{c}4.73(0.97) \\
{[15]}\end{array}$ \\
\hline $\begin{array}{l}2^{\circ} \\
\text { Estádio }\end{array}$ & $\begin{array}{c}2.89(0.13) \\
{[66]}\end{array}$ & $\begin{array}{c}4.36(0.93) \\
{[107]}\end{array}$ & $\begin{array}{c}2.66(0.16) \\
{[9]}\end{array}$ & $\begin{array}{c}5.20(0.55) \\
{[15]}\end{array}$ & $\begin{array}{c}4.80(0.48) \\
{[5]}\end{array}$ & $\begin{array}{c}5.00(0.46) \\
{[13]}\end{array}$ \\
\hline $\begin{array}{l}3^{\circ} \\
\text { Estàdio }\end{array}$ & $\begin{array}{c}3.31(0.13) \\
{[42]}\end{array}$ & $\begin{array}{c}6.55(0.31) \\
{[85]}\end{array}$ & $\begin{array}{c}3.66(0.23) \\
{[9]}\end{array}$ & $\begin{array}{c}7.92(0.66) \\
{[12]}\end{array}$ & 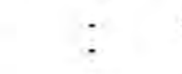 & $\begin{array}{c}10.20(1.74) \\
{[5]}\end{array}$ \\
\hline $\begin{array}{l}4^{\circ} \\
\text { Estádio }\end{array}$ & $\begin{array}{c}10.16(0.40) \\
{[43]}\end{array}$ & $\begin{array}{c}15.12(0.77) \\
{[50]}\end{array}$ & $\begin{array}{c}13.33(1.09) \\
{[9]}\end{array}$ & $\begin{array}{c}18.09(0.79) \\
{[11]}\end{array}$ & : & $\begin{array}{c}18.50(1.65) \\
{[4]}\end{array}$ \\
\hline $\begin{array}{l}\text { Estágio } \\
\text { Pupal }\end{array}$ & $\begin{array}{c}5.32(0.21) \\
{[79]}\end{array}$ & $\begin{array}{c}6.71(0.43) \\
{[32]}\end{array}$ & $\begin{array}{c}5.25(0.75) \\
{[8]}\end{array}$ & $\begin{array}{c}6.05(0.47) \\
{[17]}\end{array}$ & $\begin{array}{c}7.33(1.45) \\
{[3]}\end{array}$ & $\begin{array}{c}6.80(0.86) \\
{[5]}\end{array}$ \\
\hline $\begin{array}{l}1^{\circ}+2^{\circ} \\
\text { Estádios }\end{array}$ & $\begin{array}{c}5.66(0.22) \\
\text { s } \quad[74]\end{array}$ & $\begin{array}{c}7.13(0.23) \\
{[94]}\end{array}$ & $\begin{array}{c}4.66(0.23) \\
{[9]}\end{array}$ & $\begin{array}{c}7.64(0.58) \\
{[17]}\end{array}$ & $\begin{array}{c}9.20(1.77) \\
{[5]}\end{array}$ & $\begin{array}{c}10.00(1.43) \\
{[13]}\end{array}$ \\
\hline $\begin{array}{l}1^{\circ}>3^{\circ} \\
\text { Estádios }\end{array}$ & $\begin{array}{cc} & 8.63(0.20) \\
\mathrm{s} & {[49]}\end{array}$ & $\begin{array}{c}13.50(0.47) \\
{[69]}\end{array}$ & $\begin{array}{c}8.33(0.23) \\
{[9]}\end{array}$ & $\begin{array}{c}16.58(0.71) \\
{[12]}\end{array}$ & - & $\begin{array}{c}26.20(1.93) \\
{[5]}\end{array}$ \\
\hline $\begin{array}{l}1^{\circ}>4^{\circ} \\
\text { Estádios }\end{array}$ & $\begin{array}{l}20.95(0.48) \\
\text { s } \quad[98]\end{array}$ & $\begin{array}{c}28.83(1.11) \\
{[53]}\end{array}$ & $\begin{array}{c}21.66(0.99) \\
{[12]}\end{array}$ & $\begin{array}{c}35.33(1.18) \\
{[18]}\end{array}$ & $\begin{array}{c}36.37(1.79) \\
{[8]}\end{array}$ & $\begin{array}{c}40.72(2.05) \\
{[11]}\end{array}$ \\
\hline $\begin{array}{l}1^{\circ}>4^{\circ} \\
+ \text { Pupa }\end{array}$ & $\begin{array}{c}25.67(0.55) \\
{[80]}\end{array}$ & $\begin{array}{c}34.75(1.52) \\
{[36]}\end{array}$ & $\begin{array}{c}26.62(1.61) \\
{[8]}\end{array}$ & $\begin{array}{c}41.23(1.26) \\
{[17]}\end{array}$ & $\begin{array}{c}42.66(5.54) \\
{[3]}\end{array}$ & $\begin{array}{c}45.80(3.81) \\
{[5]}\end{array}$ \\
\hline
\end{tabular}

* = Média (Erro Padrāo)

[número] 
$\left.3.79^{-14}\right)$.

A duração média do período de desenvolvimento do estágio larval de $T$. $h$. haemorrhoidalis, para larvas criadas no ambiente Laboratório foi de $21 \pm 0.5$ dias com uma dieta de presas, e de $28.8 \pm 1.1$ dias com uma dieta de ração. Esta diferença entre as duas dietas é muito significante (teste-t NS $1^{\circ} \Rightarrow 4^{\circ}$ est. $\left.=3.77^{-8}\right)$. A duração do $1^{\circ}$, $2^{\circ}, 3^{\circ}$, e $4^{\circ}$ estádios, para larvas alimentadas com presas, foi de $2.9 \pm$ $0.1,2.9 \pm 0.1,3.3 \pm 0.1$ e $10.2 \pm 0.4$ dias, respectivamente, e para larvas alimentadas com ração foi de $3.0 \pm 0.1$, $4.4 \pm 0.9,6.6 \pm 0.3$ e $15.1 \pm 0.8$ dias, respectivamente (Fig. 1). Não houve diferença significativa na duração do $1^{\circ}$ estádio entre as duas dietas mas a duração dos outros estádios tiveram diferenças muito significativas (teste- $\mathrm{t}$ NS $1^{\circ}$ est. $=0.76 ; 2^{\circ}$ est. $=1.7^{-7}$; $3^{\circ}$ est. $=5.1^{-8} ; 4^{\circ}$ est. $\left.=5.1^{-7}\right)$.

Nas larvas de $T . h$. haemorrhoidalis criadas no ambiente Escuro, a duração média do período de desenvolvimento do estágio larval foi de $21.7 \pm 1.0$ dias para as larvas alimentadas com presas, e de $35.3 \pm$ 1.2 dias para larvas alimentadas com ração. Esta diferença entre as duas dietas é muito significante (teste-t NS $1^{\circ} \Rightarrow 4^{\circ}$ est. $\left.=6.39^{-9}\right)$. A duração do $1^{\circ}$, $2^{\circ}, 3^{\circ}$ e $4^{\circ}$ estádios, para larvas alimentadas com presas, foi de $2.9 \pm$ $0.63,2.6 \pm 0.16,3.6 \pm 0.23$ e $13.3 \pm$ 1.09 dias, respectivamente, e para larvas alimentadas com ração foi de $2.9 \pm 0.25,5.2 \pm 0.55,7.9 \pm 0.66 \mathrm{e}$ $18 \pm 0.79$ dias, respectivamente (Fig. 2). Não houve diferença significativa na duração do primeiro estádio entre as duas dietas (teste-t NS $1^{\circ}$ est. $=0.96$ ). Para o $2^{\circ}, 3^{\circ}$ e $4^{\circ}$ estádio o período de

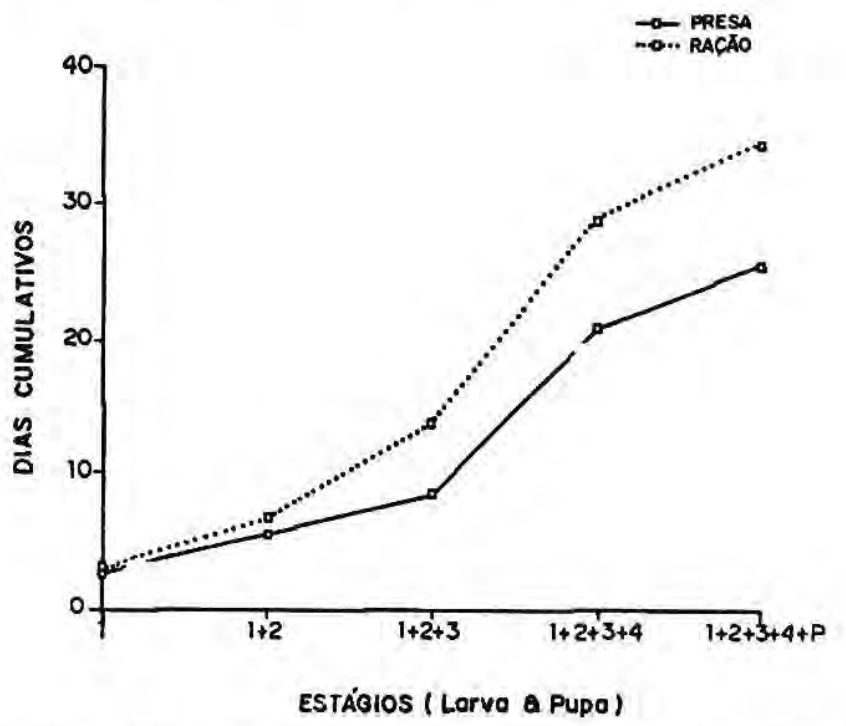

Figura 1. Período de desenvolvimento (dias-cumulativos) dos estágios imaturos de Toxorhynchites $h$. haemorrhoidalis criados no ambiente Laboratório com dietas diferentes (ração e presas), com luz e temperatura entre $24^{\circ}-30^{\circ} \mathrm{C}$. 
desenvolvimento para larvas alimentadas com presas foi mais curto em relação aos mesmos estádios alimentados com ração com uma diferença significativa (teste-t NS $2^{\circ}$ est. $=2.23^{-3} ; \quad 3^{\circ}$ est. $=4.11^{-5}$; $4^{\circ}$ est. $\left.=2.01^{-3}\right)$.

Nas larvas de $T . h$ haemorrhoidalis criadas no ambiente Frio, a duração média do período de desenvolvimento do estágio larval foi de $36.4 \pm 1.8$ dias para larvas alimentadas com presas, e de $40.7 \pm$ 2.0 dias para larvas alimentadas com ração. Esta diferença entre as duas dietas não é significante (teste-t NS $1^{\circ} \Rightarrow 4^{\circ}$ est. $\left.=0.14\right)$. A duração do $1^{\circ} \mathrm{e}$ $2^{\circ}$ estádios, para larvas alimentadas com presas, foi de $3.6 \pm 0.6$ e $4.8 \pm$ 0.5 dias, respectivamente, e para larvas alimentadas com ração foi de $4.7 \pm 1.0$ e $5.0 \pm 0.5$ dias, respectivamente (Fig. $3)$. Não houve diferenças significativas na duração dos estádios larvais entre as duas dietas quando analisadas isoladamente (teste-t NS $1^{\circ}$ est. $=0.39$; $2^{\circ}$ est. $\left.=0.81\right)$.

O tempo de desenvolvimento larval, do $1^{\circ}$ ao $4^{\circ}$ estádio, para larvas alimentadas com presas nos ambientes de Laboratćrio, Escuro e Frio, foi de $21 \pm 0,48,21 \pm 0,99$ e $36 \pm 1.79$ dias, respectivamente, sendo evidente que $o$ menor período de desenvolvimento aconteceu no ambiente Laboratório e o maior no ambiente Frio. Esta diferença entre o ambiente Frio e os ambientes Laboratório e Escuro é muito significativa (teste-t NS frio $\mathrm{X}$ lab $1^{\circ} \Rightarrow 4^{\circ}$ est. $=4.39^{-14}$; frio $\mathrm{X}$ esc $1^{\circ} \Rightarrow 4^{\circ}$ est. $=3.78^{-7}$ ). Porém, no ambiente Escuro o desenvolvimento

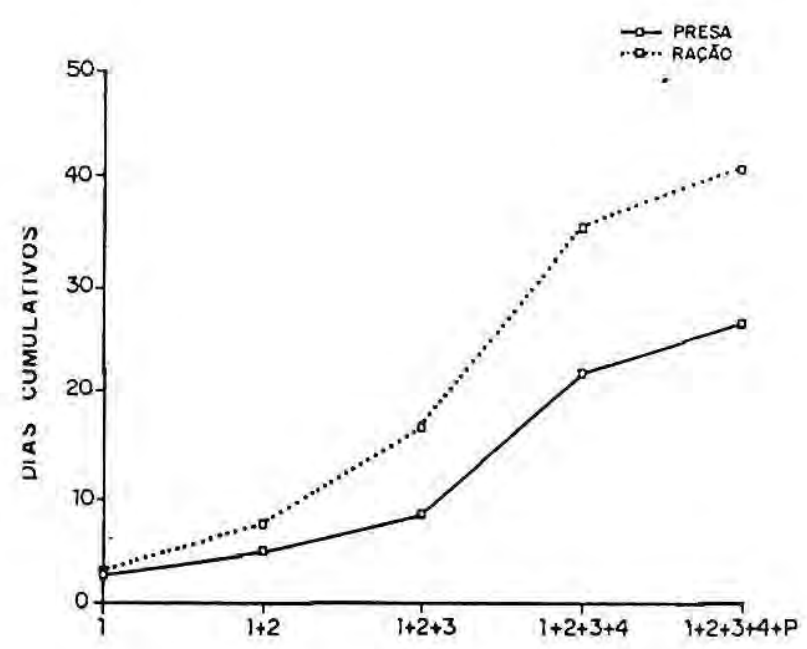

ESTÁGIOS (Larva a PUDa)

Figura 2. Período de desenvolvimento (dias-cumulativos) dos estágios imaturos de Toxorhynchites $h$. haemorrhoidalis criados no ambiente Escuro com dietas diferentes (ração e presas), sem luz e temperatura entre $28^{\circ}-31^{\circ} \mathrm{C}$. 


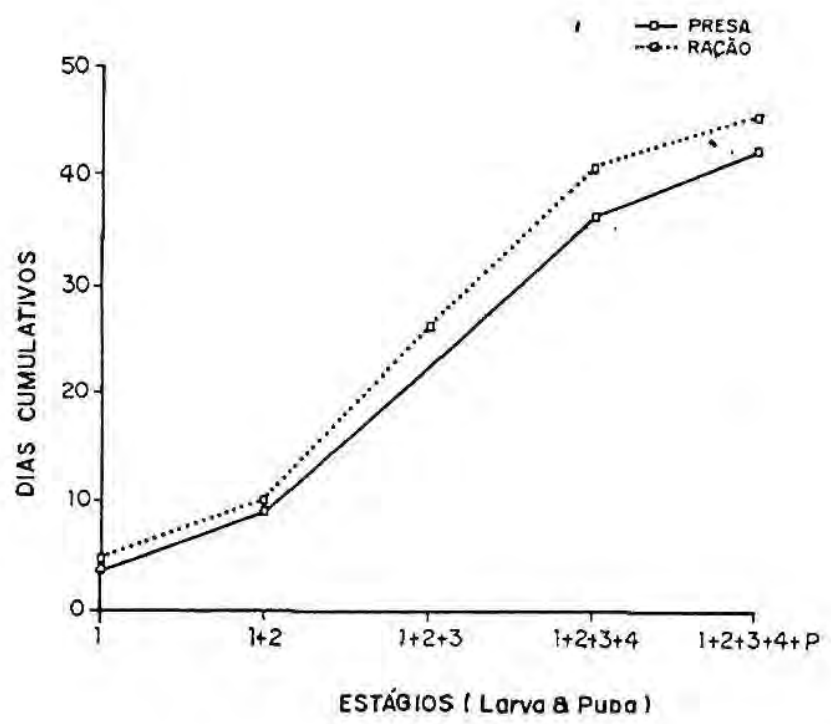

Figura 3. Período de desenvolvimento (dias-cumulativos) dos estágios imaturos de Toxorhynchites $h$. haemorrhoidalis criados no ambiente Frio com dietas diferentes (ração e presas), com luz e temperatura entre $22^{\circ}-26^{\circ} \mathrm{C}$.

não é significativamente diferente ao do Laboratório com exceção do $4^{\circ}$ estádio onde a duração no Escuro foi significativamente maior que 0 Laboratório $(13 \pm 1.09$ e $10 \pm 0.40$ dias, respectivamente: teste-t NS $1^{\circ}$ est. $=0.97 ; \quad 2^{\circ}$ est. $=0.55 ;$ $3^{\circ}$ est. $=0.26 ; \quad 4^{\circ}$ est. $=2.98^{-3}$; $1^{\circ} \Rightarrow 4^{\circ}$ est. $=0.62$ ) (Fig. 4). Quando analisados isoladamente os estádios de desenvolvimento no ambiente Laboratório são significativamente diferentes aos do ambiente Frio, somente no $2^{\circ}$ estádio $(2.9 \pm 0.13$ e $4.8 \pm 0.48$ dias, respectivamente: teste-t NS $2^{\circ}$ est. $\left.=4.92^{-4}\right)$; esta diferença no $2^{\circ}$ estádio também é significante ao comparar o ambiente Escuro com o ambiente Frio $(2.66 \pm 0.16$ e $4.8 \pm$ 0.48 dias, respectivamente: teste-t NS $2^{\circ}$ est. $\left.=2.70^{-4}\right)$.
O tempo de desenvolvimento das larvas alimentadas com ração nos ambientes Laboratório, Escuro e Frio, foi de $28 \pm 1.11,35 \pm 1.18$ e $40 \pm 2.05$ dias, respectivamente, indicando, também, um período menor de desenvolvimento para o ambiente Laboratório e maior para o ambiente Frio. Estas diferenças entre os ambientes são significantes sendo que a diferença entre o Escuro e o Frio é menos significante (teste-t NS lab X esc $=2.08^{-3} ;$ esc $X$ frio $=0.021 ;$ lab $\mathrm{X}$ frio $=2.58^{-5}$ ). A análise isolada dos estádios indica que não existem diferenças significantes entre os ambientes na duração de cada estádio larval, com exceção da diferença entre o ambiente Laboratório e Frio no $1^{\circ} \mathrm{e}$ $3^{\circ}$ estádio $\left(1^{\circ}\right.$ est. $=3 \pm 0.10,4.7 \pm$ $0.97 ; 3^{\circ}$ est. $=6.6 \pm 0.31,10.2 \pm 1.74$ 


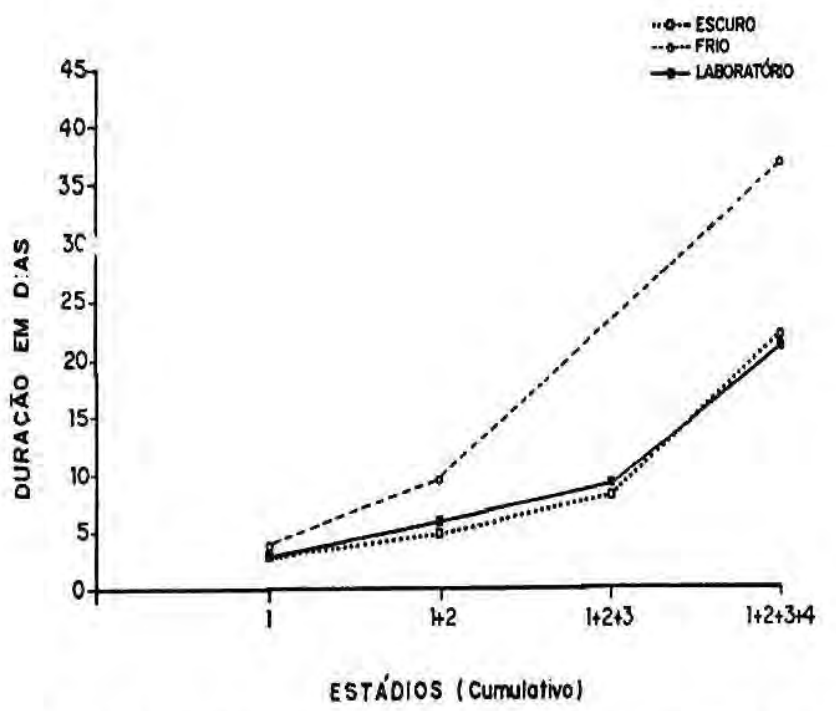

Figura 4. Período de desenvolvimento (dias-cumulativos) de larvas de Toxorhynchites $h$. haemorrhoidalis criadas no laboratório com dieta de presas em diferentes ambientes (Laboratório, Frio e Escuro).

dias, respectivamente: teste-t NS $1^{\circ}$ est. $=2.16^{-4} ; 3^{\circ}$ est. $\left.=9.05^{-3}\right)($ Fig. 5).

Os resultados indicam que larvas de $T . h$. haemorrhoidalis, criadas com dieta de ração, completam seu desenvolvimento, apesar de demorarem mais para atingir o estágio adulto. A luminosidade não teve influência no tempo de desenvolvimento das larvas alimentadas com presas, ao contrário das alimentadas com ração. A baixa temperatura influenciou o desenvolvimento das larvas, independentemente da dieta, prolongando o período de desenvolvimento.

Dados comparativos com outras espécies do gênero são resumidos na Tabela 2. TRPIS (1979) criou larvas de T. brevipalpis com ração de fígado obtendo um desenvolvimento larval de 36 dias, diferente de nossa criação que foi de 28 dias para larvas alimentadas com ração de peixe. Para as larvas alimentadas com presas, TRPIS (1979) obteve um período de 20 dias em condições similares às nossas, nas quais obtivemos um período de 21 dias. É interessante notar que a criação com presas teve basicamente a mesma duração para as duas espécies, enquanto que $T$. brevipalpis demorou oito dias a mais, com a dieta de ração. Esse fato provavelmente é decorrente de diferenças nutricionais entre as rações ou indica um maior aproveitamento da ração pelo $T$. h. haemorrhoidalis. Está evidente que o tempo médio de desenvolvimento no laboratório, para outras espécies em zonas temperadas, é menor que nas espécies tropicais. Tambem é importante registrar, que é muito difícil comparar resultados de criações feitas em condições diferentes, em virtude do grande 


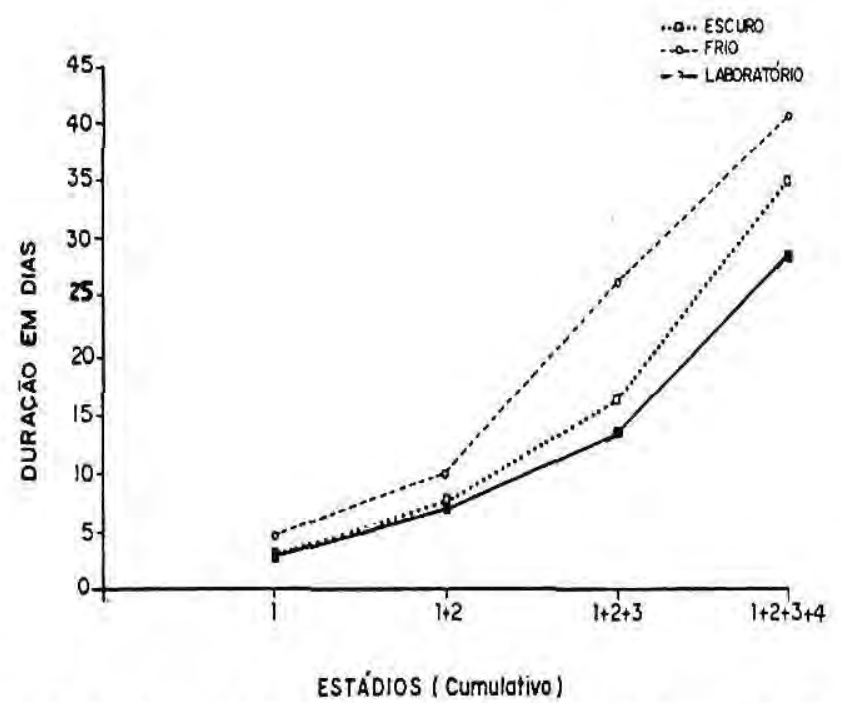

Figura 5. Periodo de desenvolvimento (dias-cumulativos) de larvas de Toxorhynchites $h$. haemorrhoidalis criadas no laboratório com dieta de ração, em ambientes diferentes (Laboratório, Frio e Escuro).

número de variáveis envolvidas que podem influenciar o desenvolvimento.

O estágio de pupa teve uma duração média de $5.32 \pm 0.21$ dias para pupas geradas a partir de larvas alimentadas com presas e de $6.71 \pm$ 0.43 dias para larvas alimentadas com ração. Esta diferença entre as duas dietas para larvas criadas em laboratório é significativa (teste-t NS Pupa $=1.64^{-3}$ ), porém, não houve uma diferença significativa entre a duração do estágio da pupa nos diferentes ambientes de criação (Tab. 1). A duração do estágio de pupa do $T$. $h$. haemorrhoidalis, para pupas geradas a partir de larvas alimentadas com presas, ficou comparativamente dentro da média das outras espécies criadas em condições similares (Tab. 2).

A emergência dos adultos ocorreu sempre durante o dia com as fêmeas emergindo antes que os machos. Dados sobre emergência em outras espécies do gênero confirmam este mesmo padrão (CORBET \& ALI, 1987; STEFFAN \& EVENHUIS, 1981).

Pelos resultados obtidos e análises realizadas, com $T$. $h$. haemorrhoidalis, pode-se concluir que: O período de desenvolvimento da espécie, em laboratório, pode ser controlado pela qualidade e quantidade de alimentação e pela manipulação das condições de luminosidade e temperatura. O período de desenvolvimento mais curto é obtido em condições de alta luminosidade, temperatura entre $26^{\circ}-30^{\circ} \mathrm{C}$ e dieta de presas ; larvas criadas com ração de peixe conseguem atingir o estágio adulto; larvas alimentadas com presas não sofreram influência da luminosidade, ao contrário de larvas alimentadas com 
Tabela 2. Tempo médio de desenvolvimento de espécies do gênero Toxorhynchites criados no laboratório.

\begin{tabular}{|c|c|c|c|c|c|c|c|c|c|c|c|}
\hline \multirow{3}{*}{ ESPECCIE } & \multicolumn{8}{|c|}{ DURAÇĀO (dias) } & \multirow{3}{*}{ ALIM } & \multirow[b]{3}{*}{ TEMP. } & \multirow[b]{3}{*}{ REFERENCIA } \\
\hline & \multirow[t]{2}{*}{ Ovo } & \multicolumn{5}{|c|}{ LARVA } & \multirow[t]{2}{*}{ PUPA } & \multirow{2}{*}{ TOTAL } & & & \\
\hline & & $1^{\circ}$ & $2^{\circ}$ & $3^{\circ}$ & $4^{\circ}$ & TOTAL* & & & & & \\
\hline T. r. rutilus & 16 & 1.2 & 26 & 3.8 & 8,0 & 15.6 & 6.0 & 21.6 & L250 & $28^{\circ} \mathrm{C}$ & FOCKS et al., 1977 \\
\hline $\begin{array}{l}T \text {. brevipalpis } \\
T \text {. brevipalpis } \\
T \text {. brevipalpis } \\
T \text {. brevipalpis } \\
T \text {. brevipalpis }\end{array}$ & & $\begin{array}{l}3.6 \\
1.7 \\
1.2 \\
1.0 \\
1.0\end{array}$ & $\begin{array}{l}4.0 \\
2.3 \\
2.2 \\
1.5 \\
1.4\end{array}$ & $\begin{array}{l}5.1 \\
2.8 \\
2.6 \\
2.4 \\
2.4\end{array}$ & $\begin{array}{r}23.5 \\
21.5 \\
13.7 \\
6.1 \\
22.1\end{array}$ & $\begin{array}{l}36.2 \\
28.2 \\
19.8 \\
11.0 \\
26.9\end{array}$ & & & $\begin{array}{l}\text { R } \\
\text { L5 } \\
\text { L10 } \\
\text { L30 } \\
\text { L8R }\end{array}$ & $\begin{array}{l}26^{\circ} \mathrm{C} \\
26^{\circ} \mathrm{C} \\
26^{\circ} \mathrm{C} \\
26^{\circ} \mathrm{C} \\
26^{\circ} \mathrm{C}\end{array}$ & $\begin{array}{l}\text { TRPIS, } 1979 \\
\text { TRPIS, } 1979 \\
\text { TRPIS, } 1979 \\
\text { TRPIS, } 1979 \\
\text { TRPIS, } 1979\end{array}$ \\
\hline$T$ amboinensis & 2.6 & 1.9 & 1.8 & 3.4 & 20.1 & 27.2 & 5.0 & 32.2 & $\mathrm{~L} 5$ & $27^{\circ} \mathrm{C}$ & STEFFAN et al., 1980 \\
\hline T. theobaldi & 2.0 & 1,3 & 2 & $4+$ & $7-12$ & & $4-7$ & & $\mathrm{~L} 20$ & $28^{\circ} \mathrm{C}$ & RUBIO et al, 1980 \\
\hline $\begin{array}{l}T \text {. towadensis } \\
T \text {. sp. (Okinawa) } \\
T . m \text { yaeyamae } \\
T \text {. amboinensis } \\
T \text {. spiendens }\end{array}$ & $\begin{array}{l}2.3 \\
2.4 \\
2.2 \\
1.8 \\
2.2\end{array}$ & $\begin{array}{l}2.1 \\
1.5 \\
1.5 \\
1.6 \\
1.6\end{array}$ & $\begin{array}{l}1.5 \\
2.2 \\
2.2 \\
1.5 \\
1.4\end{array}$ & $\begin{array}{l}2.3 \\
2.4 \\
2.4 \\
2.9 \\
2.7\end{array}$ & $\begin{array}{l}8.4 \\
8.6 \\
8.8 \\
7.1 \\
7.2\end{array}$ & $\begin{array}{l}14.3 \\
14.7 \\
14.9 \\
13.1 \\
13.9\end{array}$ & $\begin{array}{l}5.3 \\
5.2 \\
5.1 \\
6.2 \\
6.0\end{array}$ & $\begin{array}{l}19.6 \\
19.9 \\
20.0 \\
19.3 \\
18.9\end{array}$ & $\begin{array}{l}\mathrm{L} 20 \\
\mathrm{~L} 20 \\
\mathrm{~L} 20 \\
\mathrm{~L} 20 \\
\mathrm{~L} 20\end{array}$ & $\begin{array}{l}26^{\circ} \mathrm{C} \\
26^{\circ} \mathrm{C} \\
26^{\circ} \mathrm{C} \\
26^{\circ} \mathrm{C} \\
26^{\circ} \mathrm{C}\end{array}$ & $\begin{array}{l}\text { HORIO \& TSUKAMOTO, } 1985 \\
\text { HORIO \& TSUKAMOTO, } 1985 \\
\text { HORIO \& TSUKAMOTO, } 1985 \\
\text { HORIO \& TSUKAMOTO, } 1985 \\
\text { HORIO \& TSUKAMOTO, } 1985\end{array}$ \\
\hline $\begin{array}{l}\text { T. h. haemorrhoidalis } \\
T . h \text { haemorrhoidalis } \\
T \text {. h. haemorrhoidalis } \\
T \text {. h. haemorrhoidalis } \\
T \text {. h. haemorrhoidalis } \\
T \text {. h. haemorrhoidalis }\end{array}$ & & $\begin{array}{l}2.9 \\
3.0 \\
2.9 \\
2.9 \\
3.6 \\
4.7\end{array}$ & $\begin{array}{l}2.9 \\
4.4 \\
2.7 \\
5.2 \\
4.8 \\
5.0\end{array}$ & $\begin{array}{r}3.3 \\
6.6 \\
3.7 \\
7.9 \\
10.2\end{array}$ & $\begin{array}{l}10.2 \\
15.1 \\
13.3 \\
18.1 \\
18.5\end{array}$ & $\begin{array}{l}21.0 \\
28.8 \\
21.7 \\
35.3 \\
36.4 \\
40.7\end{array}$ & $\begin{array}{l}5.3 \\
6.7 \\
5.3 \\
6.1 \\
7.3 \\
6.8\end{array}$ & $\begin{array}{l}25.7 \\
34.8 \\
26.6 \\
41.2 \\
42.7 \\
45.8\end{array}$ & $\begin{array}{l}\mathrm{L} 10 \\
R \\
\mathrm{~L} 10 \\
R \\
\mathrm{~L} 10 \\
\mathrm{R}\end{array}$ & $\begin{array}{l}26^{\circ} \mathrm{C} \\
26^{\circ} \mathrm{C} \\
30^{\circ} \mathrm{C} \\
30^{\circ} \mathrm{C} \\
24^{\circ} \mathrm{C} \\
24^{\circ} \mathrm{C}\end{array}$ & $\begin{array}{l}\text { Este trabalho } \\
\text { Este trabalho } \\
\text { Este trabalho } \\
\text { Este trabalho } \\
\text { Este trabalho } \\
\text { Este trabalho }\end{array}$ \\
\hline
\end{tabular}

* eclosão até empupar

***- L\# = Larva + N\%dia

ração; a baixa temperatura influencia negativamente resultando num aumento do período de desenvolvimento.

\section{AGRADECIMENTOS}

Agradeço ao Dr. José Alberto S. Nunes de Mello pelo incentivo para o desenvolvimento deste trabalho. Um agradecimento muito especial ao Antonio Faustino Netto, Zeferino Franco da Silva, Wanilze Gonçalves Barros e Dalva Inomata Azevedo pela ajuda na coleta e criação dos mosquitos.

\section{Referências bibliográficas}

CORBET, P. S.; ALI, H. J. 1987. Diel patterns of pupation and emergence, and protogyny, in Toxorhynchites brevipalpis brevipalpis (Theobald) (Diptera,

$$
\begin{aligned}
& \text { **- eclosão até emergència } \\
& R=\text { Ração }
\end{aligned}
$$

Culicidae): a laboratory study. $A n n$. Trop. Med. Parasit., 81(4):437-443.

FOCKS, D. A.; HALL, D. W.; SEAWRIGHT, J. A. 1977. Laboratory colonization and biological observations of Toxorhynchites rutilus rutilus. Mosq. News, 37(4):751-755.

HORIO, M.; TSUKAMOTO, M. 1985. Successful laboratory colonization of three Japanese species of Toxorhynchites mosquitoes. Jpn. J. Sanit. Zool. 36(2):87-93.

HUTCHINGS, R. S. G. 1990. Observações biológicas de Toxorhynchites (Lynchiella) haemorrhoidalis haemorrhoidalis (Fabricius, 1794) (Diptera: Culicidae) numa floresta de terra-firme da Amazônia Central e no laboratório. Dissertação de Mestrado. PPG INPA/FUA. 89p.

HUTCHINGS, R. S. G. 1991. "Possibilidades do uso de espécies do gênero Toxorhynchites THEOBALD, 1901 (Diptera: Culicidae) como controladores biológicos de Culicidae na Amazônia." 
Capitulo 14, pp.383-385. Em: VAL, A.L., FIGLIUOLO, R. \& FELDBERG, E. (eds.) Bases Cientificas para Estratégias de Preservação $e$ Desenvolvimento da Amazônia: Fatose Perspectivas. Vol. I INPA, Imprensa Universitária Universidade do Amazonas, Manaus, 440p.

HUTCHINGS, R. S. G. no prelo. Palm bract breeding sites and their exploitation by Toxorhynchites (Lynchiella) haemorrhoidalis haemorrhoidalis (Diptera: Culicidae) in an upland forest of the Central Amazon. Journal of Medical Entomology.

MACHADO-ALLISON, C. E. 1981. Ecologia de los mosquitos (Culicidae).Il. Larvas y pupas. Acta Biol. Venez., 11(1):51-129. RUBIO, Y., RODRIGUEZ, D., MACHADOALLISON, C. E.; LEON, J. A. 1980. Algunos aspectos del comportamento de
Toxorhynchites theobaldi (Diptera Culicidae). Acta Cient. Venez., 31:345351.

STEFFAN, W, A. 1975. Systematics and biological control potential of Toxorhynchites (Diptera: Culicidae). Mosq. Syst., 7(1): 59-67.

STEFFAN, W. A.; EVENHUIS, N. L. 1981. Biology of Toxorhynchites. Ann. Rev. Entomol, 26:159-181.

STEFFAN, W. A., STOAKS, R. D.; EVENHUIS, N. L. 1980. Biological observations of Toxorhynchites amboinensis (Diptera: Culicidae) laboratory. J. Med. Entomol. 17(6): 515-518.

TRPIS, M. 1979. Development of the predatory larvae of Toxorhynchites brevipalpis (Diptera: Culicidac) on nonprey diet. J. Med. Entomol. 16(1): 2628.

(Aceito para publicação em 21/7/93) 